\title{
DYNAMICS OF TARGET PLASMA HEATED BY NEUTRAL BEAMS IN A MIRROR
}

Andrey A. Kabantsev

Budker Institute of Nuclear Physics

avenue Lavrent'ev, 11

630090 Novosibirsk, Russia

$+7(3832) 359550$
Vladimir G. Sokolov

Budker Institute of Nuclear Physics

avenue Lavrent'ev, 11

630090 Novosibirsk, Russia

$+7(3832) 359550$
Sergey Yu. Taskaev

Budker Institute of Nuclear Physics

avenue Lavrent'ev, 11

630090 Novosibirsk, Russia

$+7(3832) 359121$

taskaev@inp.nsk.su

\begin{abstract}
Target plasma behavior in a mirror when heated by neutral beams was studied. Considerable changes of target plasma density depending on target quality were found. Neutral beam injection resulted in a considerable decrease of target plasma density for the case of dense subsonic jet being a target. For another case (collisionless target plasma) the neutral beam injection led to increase of target plasma density.

In this paper experimental results, simulations and explanations are given.
\end{abstract}

\section{INTRODUCTION}

In experiments on plasma heating by neutral beams at a mirror system, a change in target plasma density was observed. Neutral beam injection resulted in a considerable decrease of target plasma density for the case of dense subsonic jet being a target. For another case (collisionless target plasma) the neutral beam injection led to increase of target plasma density.

An experimental layout is followed by a detailed consideration of a case of collision target plasma. Experimental results are given. The time-dependent problem of plasma flow is considered by the two-fluid magnetohydrodynamic approach with allowance for the population of hot ions in a mirror system, a numerical modeling is carried out, an adequacy between the model and the phenomenon is shown, and an explanation of the phenomenon is given. The dynamics of the potentials of an electric field, thermal force, ion pressure, and viscous force are considered in detail. An explanation is given.

Then, a collisionless target plasma case is considered.

Main conclusions are given in the last section.

\section{EXPERIMENTAL LAYOUT}

The AMBAL-Yu is a classical mirror with minimum-B [1]. The magnetic field is $0.65 \mathrm{~T}$ in the center of the device. The mirror ratio is 2 . The distance between the magnetic mirrors is 1 meter. Beams of hydrogen atoms $(16 \mathrm{keV}, 100 \mathrm{~A}, 200 \mu \mathrm{s})$ injected into the trap perpendicularly to the axis form hot-ion plasma. Before the injection, the trap was filled with a target plasma. The plasma jet was produced by an arc source, which was located outside the mirror near its end system. The plasma jet generated by this source entered the mirror cell along the magnetic field lines.

\section{COLLISION TARGET PLASMA}

Experiments in AMBAL-Yu mirror system revealed an unexpected and interesting phenomenon: a substantial decrease in the density of the target plasma jet during the injection of high-energy hydrogen atoms.

Before the injection, the device was filled with a plasma with a density of $2.4 \times 10^{14} \mathrm{~cm}^{-3}$ and with characteristic electron and ion temperatures of about $10 \mathrm{eV}$. The plasma jet was produced by an arc source with a slot discharge channel [2]. The jet was subsonic upstream and supersonic downstream from the exit side of the mirror cell. Due to the injection of atomic beams, a population of hot ions with an average energy of $6 \mathrm{keV}$ and density up to $1.1 \times 10^{13} \mathrm{~cm}^{-3}$ was generated in a volume of 31 . At the same time, the density of the target plasma jet decreased significantly (by a factor of 2.5).

\section{A. Experimental results}

The main experimental results are the following: target plasma density in the hot-ion region decreased; 
electron temperature in the mirror increased to $25 \mathrm{eV}$; the jet dimensions do not change; between the injection region and the input throat a shock wave propagating upstream is observed; the analyzers and bolometer that are located at the plasma receiver detect a substantial increase in the ion energy and heat flux. At the same time, the jet-plasma parameters measured with the probe in the upstream region from the input throat do not change noticeably. Plasma source operation does not change. Ion-cyclotron radiation does not grow. Since the injected beams are symmetrical with respect to the axis, the momentum transfer from the neutral beams to the target plasma does not play an important role.

\section{B. First explanations}

Here is one of the first explanations of the phenomenon. A cold ion is replaced by a hot one with a larger Larmor radius due to charge-exchange of a high energy atom on a cold ion. Therefore, the density of ions increases on the periphery and decreases in the center. As the end is a conductor the electrons in the center flow out of the mirror along the magnetic field lines, and on the periphery they flow inside. The development of two-flow instability and the transport of plasma across the magnetic field lines are possible. But the measurements carried out with a cesium low-energy neutral particles analyzer [3] did not show any significant transverse plasma flow.

Another variation of the explanation is the following. As hot ions in the occupied area heat electrons directly due to collisions, than subsonic electron flow is accelerated. The electrons leave the hot ion area more quickly, and the flow from the source is not changed. A longitudinal electric field appears at the boundary that accelerates the ions (note that the longitudinal electric field here has another sign according to the right model described below). The plasma flow is accelerated, the plasma density decreases. But this model does not explain many experimental results.

\section{Numerical model}

An understanding came when the plasma jet was considered within the framework of magneto hydrodynamics $[4,5]$. The set of equations includes the continuity, motion, and heat flux equations. Timedependent problem of plasma flow by the two-fluid magnetohydrodynamics approach with allowance for the population of hot ions in a mirror was considered [6]. Numerical calculations were made. The time-dependent solution for the plasma jet parameters on axis was determined. Good agreement was found between the results of simulations and all experimental data. The dynamics of the potentials are considered in detail [7].
1. Set of equations. We will consider the plasma flow moving along the inhomogeneous magnetic field lines within the framework of two-fluid magnetic hydrodynamics. We will seek a time-dependent solution for the plasma-jet parameters on the axis, provided that the high-energy ion population appears in the local volume.

We assume that the fully ionized plasma contains only protons and electrons. We suppose that the ion flow velocity is equal to electron flow velocity. A substantial target-plasma density drop takes place when the hot-ion density is only about $1 \%$ of the jet-plasma density. Hence, we assume that the target plasma ion density is equal to the electron density everywhere in the plasma volume including in the hot-ion region. We assume $\partial \mathbf{B} / \partial \mathrm{t}=0$, neglecting the magnetic field variation which appears due to the presence of plasma. We use a coordinate system with the z-axis aligned with the magnetic field lines. We write the velocity vector as $\mathbf{u}=u \mathbf{B} / \mathrm{B}$. We neglect the electron inertia. The plasma source is located at a boundary.

The set of equations including the continuity, motion, and heat flux equations for both protons and electrons can be written in the following form:

$$
\begin{aligned}
& \frac{\partial n}{\partial t}+B \frac{\partial}{\partial z}\left(\frac{n u}{B}\right)=0, \\
& M n\left(\frac{\partial u}{\partial t}+u \frac{\partial u}{\partial z}\right)=-\frac{\partial p}{\partial z}-\frac{\partial \pi_{i z z}}{\partial z},
\end{aligned}
$$

$\frac{\partial}{\partial t}\left(\frac{M n}{2} u^{2}+\frac{3}{2} p_{i}\right)+$

$B \frac{\partial}{\partial z}\left\{\frac{1}{B}\left(\left(\frac{M n}{2} u^{2}+\frac{5}{2} p_{i}+\pi_{i z z}\right) u-\chi_{\|}^{i} \frac{\partial T_{i}}{\partial z}\right)\right\}=Q_{i}$

$\frac{\partial}{\partial t}\left(\frac{3}{2} p_{e}\right)+B \frac{\partial}{\partial z}\left\{\frac{1}{B}\left(\frac{5}{2} p_{e} u-\chi_{\|}^{e} \frac{\partial T_{e}}{\partial z}\right)\right\}=Q_{e}$.

Pressure is $p=p_{i}+p_{e}$, where $p_{i}=n T_{i}$ and $p_{e}=n T_{e}$. The viscosity stress tensor component is written as $\pi_{\mathrm{izz}}=-\eta_{0}{ }^{\mathrm{i}} \mathrm{W}_{\mathrm{zz}}, \quad \eta_{0}{ }^{\mathrm{i}}=0.96 \mathrm{n} \mathrm{T}_{\mathrm{i}} \tau_{\mathrm{i}}$, $\mathrm{W}_{\mathrm{zz}}=2 \partial \mathrm{u} / \partial \mathrm{z}-(2 / 3) \mathrm{B} \partial(\mathrm{u} / \mathrm{B}) / \partial \mathrm{z}$, where $\tau_{\mathrm{i}}$ is ion collision time. On the right-hand side of the energytransfer equations (3) and (4), the Q-terms denote the heat sources: $Q=Q_{e i}+Q_{\text {hot }}+Q_{F R}$. Here, $Q_{e i}$ is the heat source due to electron-ion collisions: $Q_{e i}{ }^{i}=-Q_{e i}{ }^{e}=3(m / M) n\left(T_{e}-T_{i}\right) / \tau_{e}$, where $\tau_{e}$ is electron collision time. $\mathrm{Q}_{\mathrm{FR}}$ is the heat source due to the work done by the electron pressure force: $Q_{F R}{ }^{i}=-Q_{F R}{ }^{e}=$ $-\mathrm{u}\left(\partial \mathrm{p}_{\mathrm{e}} / \partial \mathrm{z}\right)$. The electron and ion heat sources due to collisions with hot ions are: $\mathrm{Qhot}^{\mathrm{e}}=\mathrm{n}_{\mathrm{hot}} \mathrm{E}_{\mathrm{hot}} / \tau_{\mathrm{E}}{ }^{\mathrm{e}}$, Qhot ${ }^{\mathrm{i}}=\mathrm{n}_{\text {hot }} \mathrm{E}_{\text {hot }} / \tau_{\mathrm{E}}{ }_{\mathrm{E}}^{\mathrm{i}}$, where $\tau_{\mathrm{E}}{ }^{\mathrm{e}}$ and $\tau_{\mathrm{E}}{ }^{\mathrm{i}}$ are the times of the collisional energy transfer between the hot ions and the jet electrons and ions, respectively. 
The initial conditions describe the plasma-jet flow in the absence of the hot ions. The jet is subsonic upstream and supersonic downstream from the exit side of the mirror. In the case of steady_plasma flux emerging from the plasma source with fixed electron and ion temperatures, we write the boundary conditions near the plasma source as follows: $T_{e}=$ const, $T_{i}=$ const, $\mathrm{nu}=$ const, and $\partial \mathrm{n} / \partial \mathrm{z}=0$.

2. Numerical results. Fig. 1 shows the magnetic field and the hot-ion density distributions along the z-axis that were used in the calculations. The plasma source is located at $\mathrm{z}=0$. We assumed the hot-ion density to grow linearly during $10 \mu$ s up to $2 \times 10^{12} \mathrm{~cm}^{-3}$ and then to be constant in time. The hot-ion energy was $E_{h o t}=5 \mathrm{keV}$. Fig. 2 shows the solution of the time-dependent problem. The simulation results agree well with all experimental results.

\section{$\mathrm{B}, \mathrm{T}$}

$\mathrm{n}_{\text {hot, }}$, arb.units

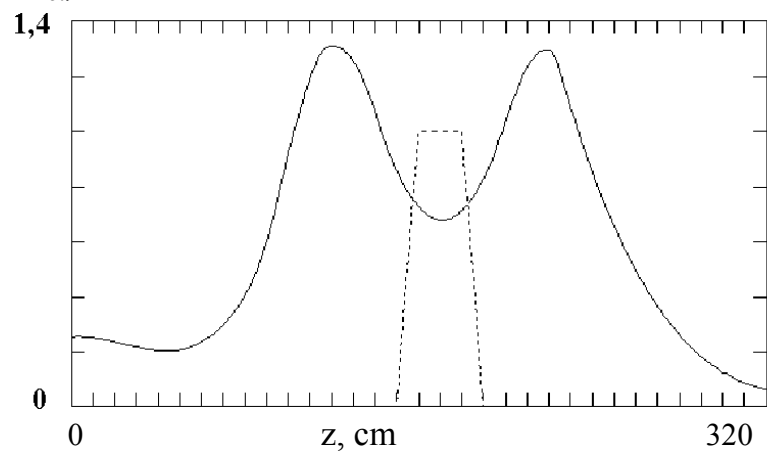

Fig. 1. Magnetic field (solid line) and hot-ion density (dashed line) distributions along the z-axis used in calculations.

D. Discussion of the time evolution of the potentials

The phenomenon appeared to be explained more clearly in terms of potentials.

The injection of atomic beams leads to a rapid accumulation of hot ions. Hot ions rapidly lose their energy in Coulomb collisions with electrons and ions of the target plasma. For example, the time during which hot ions are decelerated by the plasma, which is still dense and cold in the initial stage of injection, is equal to $3 \mu \mathrm{s}$. Most of the energy of the hot ions is converted into the energy of the plasma electrons, and only a small fraction of this energy is transferred to the ions of a target plasma. As a result, the electron temperature increases rapidly within $10 \mu \mathrm{s}$.

The heated electrons are trying to escape from the mirror cell, and plasma potential in the mirror increases significantly and confines these heated electrons in the mirror. As a result, a longitudinal electric fields arises,
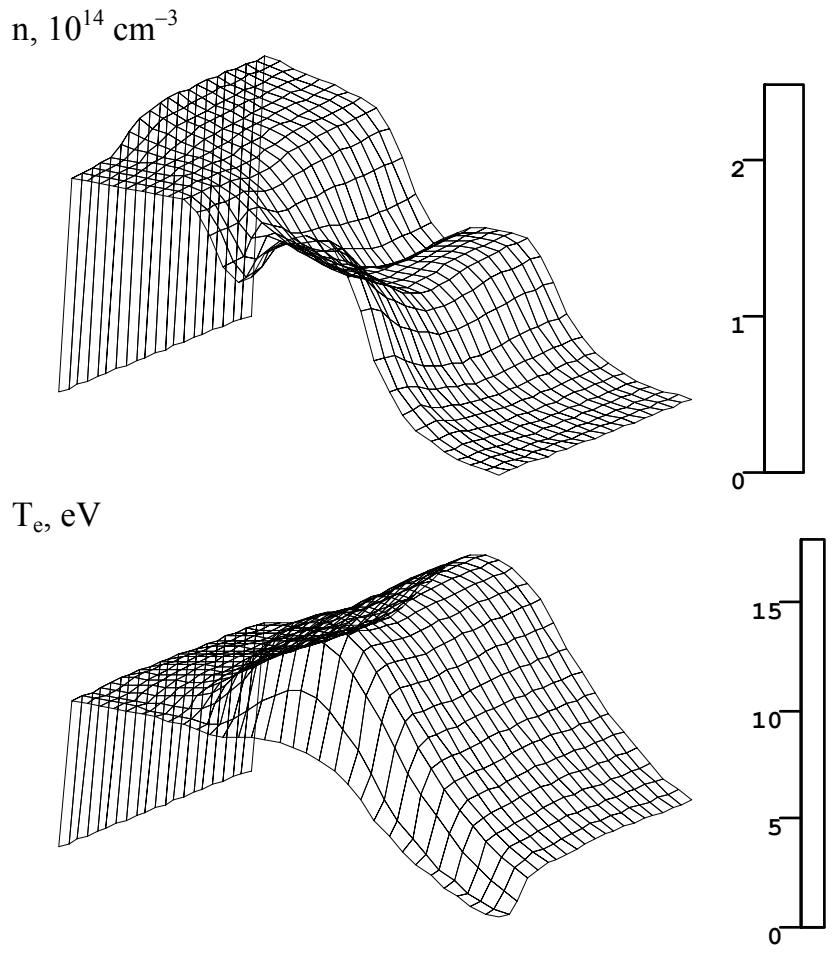

$\mathrm{T}_{\mathrm{i}}, \mathrm{eV}$
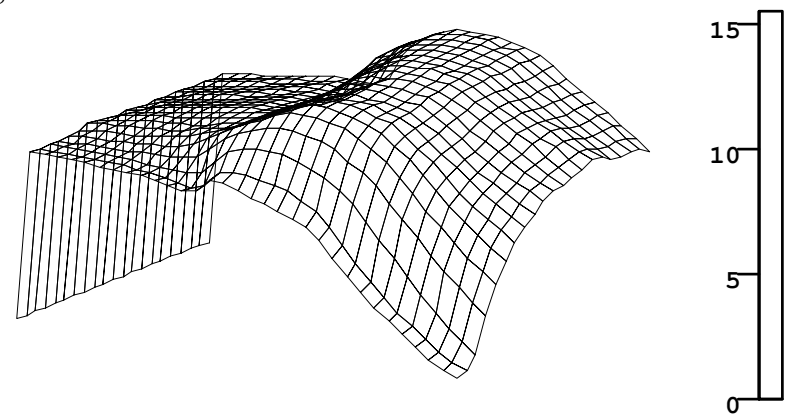

$\mathrm{u}, 10^{6} \mathrm{~cm} \mathrm{~s}^{-1}$
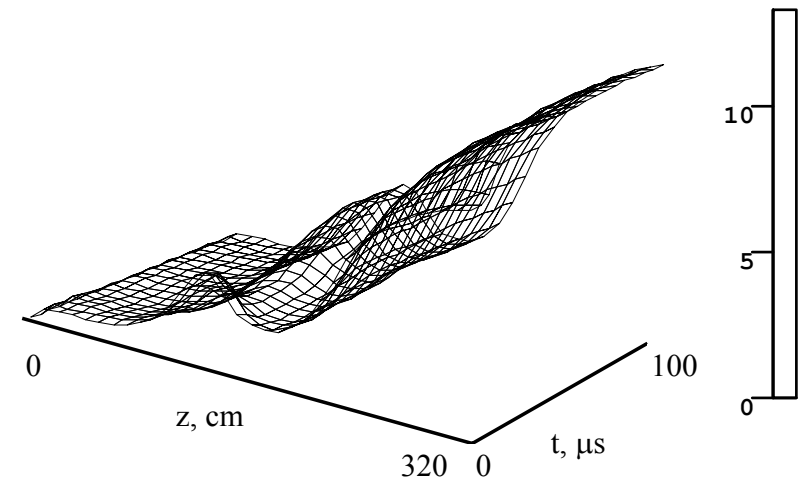

Fig. 2. The calculated isometric profiles of evolution of spatial distribution of plasma parameter on axis.

which decelerates the ions that enter the mirror cell and accelerates the ions that escape from this cell. Because of the ion deceleration, the plasma flow through the mirror 
decreases. Consequently, since the flow velocity does not change, the plasma density begins to decrease. Because the ions are decelerated in the region in front of the injection region, the deceleration of the plasma flow results in an increase in the plasma density. On the other hand, the flux of the ions that are accelerated behind this region overtakes the flux of the ions that were accelerated earlier and whose acceleration rate was lower; consequently, the plasma flow behind this region increases. After $100 \mu \mathrm{s}$, the plasma flow becomes the same as it was at the initial instant. This state of the flow will be referred to as the steady state. Our aim here is to study the corresponding density evolution.

First, we analyze the processes that occur in the zone between the plasma source and the injection region. The plasma flow is governed by the motion of plasma ions. Recall that the plasma potential in the mirror increases significantly so that electrons whose heating rate is high are confined by the corresponding longitudinal electric field, which decelerates the ions that enter the mirror cell. Consequently, the plasma density starts to increase. Since the electron thermal conductivity is high, the electron temperature increases in front of the mirror cell. The ion temperature also increases due to electron-ion collisions. The plasma potential profile becomes smooth: in the zone between the plasma source and the injection region, both the plasma density and electron temperature increase, and within the mirror cell, electrons can be confined in a relatively shallow potential well. However, in the steady state, the value of the plasma potential in the mirror exceeds the corresponding value before the injection; i.e., the ions that enter the mirror cell are, as before, decelerated by the electric field. In the steady state, the plasma flow again becomes the same as it was at the initial instant. This raises the question as to why the plasma density decreases. At first glance, the plasma density should increase, because the plasma flow is decelerated by the electric field.

We take into account a thermal force that is driven by the longitudinal gradient of the electron temperature. The effect of the electrons that escape from the region in which the temperature is higher on the plasma ions is weaker that of the electrons that escape from the region where the temperature is lower. The reason is that the collision time depends strongly on the electron velocity, $\tau \sim \mathrm{v}^{3}$. Although the momentum acquired by the ions due to their collisions with hotter electrons is higher than that acquired due to collisions with colder electrons, in the latter case, the collisions are more frequent. Consequently, the thermal force accelerates the ions that enter the mirror. However, at the center of the mirror, the sum of plasma potential and thermal force potential in the initial stage is equal to that in the steady state. Consequently, the flow velocity and, thus, the plasma density remain unchanged.
The only effect is the work produced by the ionpressure forces. In the steady state, the ion-pressure potential $\left(\varphi_{p}=\int \frac{1}{n} \frac{\partial p_{i}}{\partial z} d z\right)$ is lower than that in the initial stage. Consequently, in the steady state, the flow velocity is higher and the plasma density is lower than in the initial stage.

In order to better understand the work produced by pressure forces, we consider the behavior of the ions that escape from the mirror cell. An increase in the plasma potential, which ensures electron confinement in the mirror cell, causes an increase in the gradient of the ambipolar potential at the exit side of the mirror cell. The electric field (but not only this field) accelerates ions more strongly and takes them away from the mirror. The ions leave the mirror more quickly. At the same time, the plasma flow through the mirror decreases, which results in a substantial decrease in the density within the mirror cell. When the plasma flow becomes the same as it was in the initial stage, the drop in the total potential (the sum of potentials of the electric field, thermal force, ion pressure and viscous force) between the plasma source and the mirror cell increases, which is associated with a low plasma density within the mirror cell. Since the acceleration rate of the ions that enter the mirror cell is higher, the flow velocity of these ions increases, and, consequently, the density of these ions decreases.

\section{E. Resume}

Observations and calculations concerning this phenomenon can be concluded in the following way.

i). Injected hot ions heat efficiently plasma jet ions not directly but through electrons.

ii). Heating leads to the increase of the flow velocity of a plasma jet and accordingly to the decrease of plasma density. Work produced by ion-pressure forces exerts primary influence on decrease of the plasma density. The effect resembles the well-known effect of one-fluid hydrodynamics - the heating of a subsonic jet leads to its acceleration.

iii). In order to confine the heated electrons in a mirror system, the ambipolar potential increases. An increase in the gradient of this potential causes an increase in the acceleration rate of ions that escape from a mirror cell. The ion escape occurs mainly under the action of the electric field. At the initial moment of hot ion accumulation the increase of the ambipolar potential leads to the repulsion of input ions, a reduction of plasma flow, and a decrease in plasma density.

iiii). To study the processes under consideration, it is important to take into account the longitudinal thermal force.

IV. COLLISIONLESS TARGET PLASMA 
In the following experiments, another scenario (method) of obtaining of target plasma was used to achieve the higher electron temperature. The arc source with a ring discharge channel was used [8]. A thermal barrier in the throat [9] and an ambipolar potential in the trap were realized. Before the injection, the mirror was filled with plasma with a density of $3 \times 10^{13} \mathrm{~cm}^{-3}$, ion temperature of about $600 \mathrm{eV}$, and electron temperature of about $50 \mathrm{eV}$ [10]. High ion temperature is determined by Kelvin-Helmholtz instability heating [11]. Due to the injection of atomic beams, a population of hot ions was generated. At the same time, the density of the target plasma increased up to $10 \%$.

The temperature of ions of target plasma was determined by the balance between ion heating due to the development of Kelvin-Helmholtz instability and the energy losses due to drag on the electrons. Due to the injection of atomic beams, the hot ions apparently heated the electrons by Coulomb collisions. The electron temperature increased and consequently the temperature of target plasma ions also increased. Temperature rise for the target plasma ions resulted in an increase of characteristic collision time and increase of ion lifetime in the trap. The confinement of collisionless ions was improved while heating.

This situation of collisionless target plasma took place in the famous experiments in the 2XIIB machine [12] and so the strong effect of decrease of plasma density which we detected could not show itself, and they did not notice any increase of target plasma density.

\section{CONCLUSION}

The dynamics of target plasma heated by neutral beams in a mirror was studied and explanations were given.

In the case of a sufficient dense subsonic jet being the target plasma upon heating by neutral beams the target plasma density decreased significantly. The effect resembled the well-known effect of one-fluid hydrodynamics - the heating of a subsonic jet was found to lead to its acceleration and accordingly to the decrease of density. A significant influence of longitudinal electric field and longitudinal thermal force was revealed. The way of heating was determined to be able to differ from neutral beams. The power was not necessarily put directly into ions.

In the case of a collisionless target plasma upon heating by neutral beams, an opposite effect took place an increase of plasma density. The increase of target plasma density was determined to be related to the improvement of the confinement of collisionless ions while heating.

REFERENCES
1. E. D. Bender, V. E. Chupriyanov, G. I. Dimov, et. al., "The AMBAL-U experiment," Proc. Int. School of Plasma Physics, Villa Monastero, Varenna (Italy), p. 157, (1990).

2. G. I. Dimov, A. A. Kabantsev, and S. Yu. Taskaev, "The target plasma source with a gap shape of the gasdischarge channel for AMBAL-Yu," Voprosy Atomnoi Nauki $i$ Tekhniki, Ser.: Termoyadernyi Sintez (Problems of Atomic Science and Technology), (Nuclear Fusion), 3, 58 (1989).

3. V. G. Dudnikov, G. I. Fiksel', and S. Yu. Taskaev, "Cesium low-energy neutral particles analyzer," Fiz. Plasmy, 20, 199 (1994) [Sov. J. Plasma Phys. (Engl. Transl.), 20, 183 (1994)].

4. S. I. Braginskii, Voprosy Teorii Plasmy (Problems of Plasma Theory), Moscow: Atomizdat, 1, 183 (1963).

5. J. M. Dawson, and M. Uman, "Heating a plasma by means of magnetic pumping," Nucl. Fusion, 5, 242 (1965).

6. A. A. Kabantsev, V. G. Sokolov, and S. Yu. Taskaev, "Decrease in the Target Density during Neutral-Beam Injection into a Tandem Mirror," Fiz. Plasmy, 21, 775 (1995) [Plasma Phys. Reports (Engl. Transl.), 21, 735 (1995)].

7. S. Yu. Taskaev, "Dynamics of the Potential of a Plasma Jet Heated by Atomic Beams in a Mirror System," Fiz. Plasmy, 23, 1123 (1997) [Plasma Phys. Reports (Engl. Transl.), 23, 1042 (1997)].

8. G. I. Dimov, A. A. Ivanov, and G. V. Roslyakov, Fiz. Plasmy, 8, 970 (1982) [Sov. J. Plasma Phys. (Engl. Transl.), 8, 546 (1982)].

9. G. I. Dimov, A. A. Kabantsev, S. V. Kuzmin, et. al., "Thermal insulation of the target plasma in the "AMBAL-YU" mirror device," Fiz. Plasmy, 19, 350 (1993) [Sov. J. Plasma Phys. (Engl. Transl.), 19, 180 (1993)].

10. E. V. Gilev, G. I. Dimov, A. A. Kabantsev, et. al., "Production of hot plasmas in a mirror system by means of a gas-discharge arc source," JETP Lett., 57, 639 (1993).

11.A. A. Kabantsev, and S. Yu. Taskaev, "Ion temperature in a plasma jet," Fiz. Plasmy, 18, 635 (1992) [Sov. J. Plasma Phys. (Engl. Transl.), 18, 331 (1992)].

12.F. H. Coensgen et al. Proc. VI Int. Conf. Plasma Phys. And Contr. Nucl. Fusion Res., Berchtesgaden, 1976; Nucl. Fusion Suppl. 3, 135 (1977). 